\title{
Effects of Transcranial Direct Current Stimulation and Peripheral Stimulation on Electrical Activity of the Tibialis Anterior Muscle and Balance in Stroke Survivors with Hemiparesis - Randomized, Sham-Controlled, Double-Blind, Clinical Trial
}

\author{
Aline Marina Alves Fruhauf*, Fabiano Politti, David Correa Alves, Camila Cardoso da Silva, João Carlos \\ Ferrari Corrêa and Fernanda Ishida Corrêa
}

Postgraduate Program in Rehabilitation Sciences, Universidade Nove de Julho, Brazil

*Corresponding author: Aline Marina Alves Fruhauf, Postgraduate Program in Rehabilitation Sciences, Universidade Nove de Julho: Rua Adolpho Pinto 109. Barra Funda, São Paulo, Brazil

\section{ARTICLE INFO}

Received: 蔧 February 05, 2020

Published: 幽 February 12, 2020

Citation: Aline Marina Alves Fruhauf, Fabiano Politti, David Correa Alves, Camila Cardoso da Silva, João Carlos Ferrari Corrêa, Fernanda Ishida Corrêa. Effects of Transcranial Direct Current Stimulation and Peripheral Stimulation on Electrical Activity of the Tibialis Anterior Muscle and Balance in Stroke Survivors with Hemiparesis - Randomized, ShamControlled, Double-Blind, Clinical Trial. Biomed J Sci \& Tech Res 25(3)-2020. BJSTR. MS.ID.004219.

Keywords: Hemiparesis; Tibialis Anterior; Transcranial Direct Current Stimulation; Peripheral Electrical Stimulation; Electromyography; Balance

\section{ABSTRACT}

Objective: Lower limb motor dysfunction and lack of balance is one of the most common and disabling sequelae affecting stroke. Objectives: Analyze the effects of the combination of Transcranial Direct Stimulation (tDCS) with peripheral stimulation (PES) on the activity of the tibialis anterior (TA) paretic and balance of hemiparetic post stroke.

Methods: Participated 36 hemiparetic chronic. The TA was evaluated with EMG by median frequency (MDF)/ root mean square (RMS) and balance by Mini-BES Test. Evaluations were performed three times: pre, post 10 treatment sessions and 30 followup days. To treatment, subjects were randomized in 3 groups: PESa (active) /tDCSp (placebo); PESa /tDCSa and PESp /tDCSa. tDCS anode electrode was applied over injured motor cortex (C1/C2) and cathode uninjured (C1/C2) for 20 min associated with active dorsiflexion and PES for 30 min (active PES was applied over paretic TA and placebo in TA bone portion). The treatment was performed five times a week/ 2 week.

Results: There was no difference in RMS for either group. Intragroup MDF significantly decreased ( $\mathrm{p}<0.05$ Repeated measures ANOVA) after 10 days of treatment and follow-up in all groups; intergroup MDF was significantly lower after 10 days and follow-up in the PESa/tDCSa and PESp /tDCSa groups compared to PESa/tDCSp $(\mathrm{p}<0.05$ Repeated measures ANOVA). Balance improved significantly ( $p=0.00$ Friedman) and clinically important ( $>3$ points) in PESa /tDCSa after 10 days and follow-up there was no difference ( $p>0.05$ Kruskal-Wallis) between groups.

Conclusion: EMG, ETCC did not potentiate PES. In Balance, the combined techniques provided clinical improvement.

\section{Introduction}

Stroke often leads to a reduction in the activation of nerve pathways involved in somatosensory processing and the execution of motor actions, resulting in impaired motor control [1]. Among other complications, this condition generally leads to reduced muscle strength associated with altered muscle tone, which is denominated paresis [2]. Hemiparesis or unilateral paresis following a stroke compromises the normal function of the joints. The limitation regarding the stabilization of the ankle (equinus) affects both static and dynamic balance [3]. Equinus is the limited upward movement of the foot due to hypotonus of the gastrocnemius and soleus muscles (triceps surae) and a reciprocal reduction or absence of strength in the tibialis anterior (TA) muscle 
[3]. This dysfunction partially impedes the transference of weight to the affected limb, which anatomically interferes with postural reactions and balance [4]. A TA muscle without paresis (reduced muscle strength due to hypertonus of its antagonist) promotes a better body support base (balance) [5]. In situations of abrupt posteroanterior movements, this muscle is capable of reversing the perturbation through torque, which restores the body to the stabilization axis [5] (ankle strategy) [6]. When the sway surpasses the capacity of the muscle for reorganization through the inverted pendulum, the TA performs dorsiflexion (step strategy), thereby avoiding a fall [6].

Studies have shown that peripheral electrical stimulation (PES) of paretic muscles, such as the TA, combined with other forms of rehabilitation can lead to functional improvements [7]. PES is a rehabilitation technique that consists of the use of a controlled, low-frequency, low-intensity, external, electrical current with the aim of depolarizing the intact motor neuron to initiate and facilitate the voluntary contraction of paralyzed muscles in order to produce movement [8]. However, this technique has limitations, as repetitive stimulation in stroke survivors can lead to a task adaptation process or a plateau in functional gains after approximately 12 months of treatment [9]. Therefore, many researchers have investigated the effects of transcranial direct current stimulation (tDCS) in stroke survivors with hemiparesis in an effort to increase cortical excitability and modulate its activity to favor motor actions $[10,11]$. TDCS is noninvasive brain stimulation involving the administration of a low-intensity, monophasic electrical current to the scalp through silicone-sponge surface electrodes soaked in saline solution. The poles of the electrodes are the anode (positive pole) and cathode (negative pole) and the effects on the neuronal membrane are due to the movement of ions between the two poles [12].

The results of studies involving the administration of tDCS to stroke survivors have shown improvements in voluntary control of the ankle [13], strength of the quadriceps muscle, static postural stability [14] and the amplitude of the motor evoked potential (MEP), thereby facilitating the learning of a particular activity [15] The combination of PES and low-intensity central stimulation (tDCS) is believed to increase the stimulus of the sensory and associative cortex, which, in turn, interacts with the motor cortex, assisting in the relearning of a given task. The concomitant stimulation of the ascending and descending pathways may increase the neuronal excitability of intact structures or the recruitment of cells near the damaged site, resulting in an improvement or increase in the functional repertoire [16]. This has been demonstrated in several studies. Kwon et al. [17] evaluated the activity of the primary motor cortex (M1) in two healthy individuals during a session of tDCS combined with PES of the wrist extensors and found an increase in M1 activity. Rizzo et al. [18] investigated the MEP of healthy young individuals following the combination of tDCS and repetitive peripheral electrical stimulation of the left median nerve and found an increase in the MEP up to 60 minutes after stimulation. Celnik et al. [19] evaluated the performance of stroke survivors on motor tasks following stimulation of a peripheral nerve and tDCS and found that the combination of the two techniques led to greater improvements compared to the use of each technique alone. Sattler et al. [20] evaluated the effects of tDCS combined with PES of the radial nerve in individuals in the subacute phase of stroke and found a significant improvement in motor function of the hand, which lasted up to one month after treatment. However, Fruhauf et al. [21] evaluated the immediate effect of tDCS combined with PES on the electrical activity of the paretic TA muscle and balance in stroke survivors in the chronic phase, but found no effect following the administration of the two techniques combined, which may have been due to the fact that the authors used only a single treatment session. Based on these findings, the interaction between central and peripheral stimulation in prolonged treatments may lead to improvements in motor function, as tDCS favors the excitation of the cortex and PES favors the activation of ascending nerve fibers. Therefore, the aim of the present study was to investigate the effects of the combined use of tDCS and PES on electrical activity of the TA muscle and balance in stroke survivors with hemiparesis.

\section{Methods}

\section{Study Design}

A randomized, placebo-controlled, double-blind, clinical trial was conducted. The primary outcome was electrical activity of the TA muscle determined using electromyography (EMG). The secondary outcome was balance determined using the Mini-Bes Test. Evaluations were performed on three occasions:

a) Pre-intervention,

b) Post-intervention (after 10 treatment sessions) and

c) Follow-up (30 days after the end of the interventions). All participants were recruited from the physical therapy clinics of Nove de Julho University, São Paulo, Brazil. The flowchart of the study is displayed in Figure 1

\section{Eligibility Criteria}

The eligibility criteria were hemiparesis resulting from a stroke currently in the chronic phase (six or more months since the stroke event) [22], weakness of the TA muscle $(>1$ and $<5$ ) [22] based on the Medical Research Council (MRC), [23] adults ( $\geq 20$ years) with ambulation with or without a gait-assistance device [22] and agreement with the terms listed in the statement of informed consent. The exclusion criteria were a positive cutoff point for cognitive impairment determined using the Mini Mental State Examination ( $<11$ points, corrected for schooling), [24] diagnosis of severe depression using the Beck Depression Inventory, [25] active mobility of the ankle less than 5 degrees [13] (determined using a goniometer), muscle stiffness in flexion or extension (based on the Ashworth scale), [26] need for orthopedic insoles, rigid orthosis or the use of botulinum toxin in the lower limbs, [22] severe visual impairment (confirmed by ophthalmological exams), [22] contraindication for use of tDCS (history of seizures, tumors 
at stimulation site and metallic implants in skull - all confirmed by medical exams), [21] skin lesion at tDCS or PES application site (visual inspection of therapist),anesthesia or hyperesthesia at tDCS or PES application site (physical evaluation of superficial sensitive using a esthesiometer), diagnosis of deep vein thrombosis (confirmed by medical exam), diagnosis of degenerative disease or polyneuropathy (confirmed by medical exam) and physical therapy or alternative therapy during the development of the study or within one month after the end of the 10 treatment sessions [22].

\section{Sample Size}

The sample size was calculated with the aid of the $G^{*}$ Power program and based on a study conducted by Sabut et al. (Surface EMG Analysis of Tibialis Anterior Muscle in Walking with PES in Stroke Subjects) [27]. Root mean square (RMS) scores were used for the calculation based on mean and standard deviation values of the experimental group before $(60 \pm 6)$ and after $(110 \pm 11)$ PES. Considering $\boldsymbol{\alpha}=\mathbf{0 . 0 5}, \boldsymbol{\beta}=0.2$ (80\% power) and an effect size of 0.94 , it was determined that 12 individuals would be needed for each group (total: 36 individuals) [22].

\section{Randomization}

The 36 participants were allocated to the different groups in a randomized, counterbalanced manner using a randomization table in Excel ${ }^{\mathrm{TM}}$ with codes for the central and stimulations (active and sham) and different combinations. ${ }^{21} \mathrm{~A}$ researcher not involved in the evaluations or treatment was responsible for the random allocation of the participants to three groups: [22]

a) Active tDCS (anode over damaged hemisphere and cathode over undamaged hemisphere) combined with active PES of the paretic TA muscle.

b) Sham tDCS (anode over damaged hemisphere and cathode over undamaged hemisphere) combined with active PES of the paretic TA muscle.

c) Active tDCS (anode over damaged hemisphere and cathode over undamaged hemisphere) combined with sham PES of the paretic TA muscle.

\section{Blinding}

The NeuroConn DC-STIMULATOR PLUS device has settings that enable the selection of the active stimulation mode or sham mode by entering codes. A researcher not involved in the treatment or evaluations programed the equipment with the code to which the patient was allocated. The type of stimulation (active or sham) was not perceptible by visual cues or the external functioning of the device. Therefore, neither the researcher who placed the equipment on the patient nor the patient was aware of which treatment he/she was receiving (double-blind study).

\section{Data Collection, Management and Analysis}

For all evaluation procedures, the participants were seated on a chair with knees flexed $90^{\circ}$ and ankles in the neutral position [21].

\section{Electromyography of Tibialis Anterior Muscle}

The data on the activity of the TA muscle were analyzed by the amplitude/power of the signal (root mean square [RMS]) and muscle fiber recruitment rate (median frequency [MDF]) collected using the EMGSYSTEM $®$ electromyograph, which consists of an A/D converter with 16 bits of resolution, six channels and wireless data transmission [21]. The signals were pre-amplified with a gain of 1000 fold and a common rejection mode ratio $>100 \mathrm{~dB}$ and filtered by a $20-450 \mathrm{~Hz}$ bandpass filter. The sampling frequency was $1 \mathrm{kHz}$. The signals were coded using routines developed in MATLAB ${ }^{\circledR}$ version R2010a (The MathWorks Inc., Natick, Massachusetts, USA) [21]. Two disposable surface electrodes (Ag/AgCl - Medical Trace $囚)$ measuring $10 \mathrm{~mm}$ in diameter were positioned on the skin (previously cleaned with $70 \%$ alcohol) in the region of the TA following the norms of Surface Electromyography for the NonInvasive Assessment of Muscles (SENIAM) [28]. The participant performed three maximum voluntary contractions of TA muscle (active dorsiflexion) for 10 seconds with verbal stimulation, with a two-to-three-minute rest period between trials, followed by five consecutive concentric (isotonic) contractions of the TA muscle three times, with a two-to-three-minute rest period between trials [21]. No previous study has been developed to determine the reliability of this equipment for the population of stroke survivors, but the instrument has demonstrated solid, effective results in the investigation of muscle activity in these patients $[29,30]$.

\section{Mini-Balance Evaluation System Test (Mini-BES Test)}

Functional balance was evaluated using Mini-BES Test, which is a battery of 14 tasks distributed among four domains:

a) Anticipatory postural adjustments (transition from sitting to standing position, standing on tips of toes and one-legged stance).

b) Postural responses (different directions of body sway [posteroanterior and later lateral]).

c) Sensory orientation (feet together on stable surface with eyes open; unstable surface with eyes open; leaning with eyes closed).

d) Stability during gait (walking with change in speed; walking with horizontal movements of the head; walking around an obstacle; turning on one's own axis; and walking with and without a dual cognitive task) [31].Each item receives a score on a four-point scale ranging from 0 (worst performance) to 3 (best performance). The maximum score is 28 points.31 The Mini-BES Test has high reliability for the evaluation of stroke survivors (ICC $>0.90$ ) [32].

\section{Determination of Potential Confounding Factors}

Depressive Symptoms: Depressive symptoms were evaluated and graded about severity using the Beck Depression Inventory (BDI) [25], which is a self-administered questionnaire composed of 21 items. Each item is scored from 0 to 3 points. The total ranges 
from 0 to 63 points and is interpreted as follows: 0 to 10 indicates the absence of depression; 11 to $18=$ mild depression; 19 to $29=$ moderate depression; and 30 to $63=$ severe depression [33]. The BDI score was determined on three occasions (pre-intervention, post-intervention and 30-day follow-up). The reliability of the BDI is 0.89 and this measure has been used in studies that have shown good clinical results [34].

\section{Evaluation for Characterization of Sample}

Fugl-Meyer Scale (FMS): The measures proposed on the FMS are based on a neurological examination and sensorimotor activity of the upper and lower limbs to identify selective activity and synergic patterns in stroke survivors. The scale consists of an accumulative numeric point system for the evaluation of six aspects: range of motion, pain, sensitivity, motor function of the upper and lower limbs and balance as well as coordination and velocity, totaling 226 points [35]. Each item is scored on a threepoint ordinal scale: 0 - not performed, 1 - partially performed and 2- completely performed. The scale has a total of 100 points for normal motor function; the maximum for the upper limb is 66 and the maximum for the lower limb is 34. The FMS also enables scoring motor impairment: less than 50 points indicates severe impairment, 50-84 = marked impairment, 85-95 = moderate impairment and 96-99 = mild impairment [35]. The scale was used in the present study for the objective characterization of the participants in terms of the degree of global and specific motor impairment of the lower limbs. The literature reports that the FMS has high reliability (ICC = 0.98 ) for the evaluation of stroke survivors [36].

Interventions: For both interventions, the participant was seated in a chair with the knees flexed at $90^{\circ}$ and the ankles in the neutral position [22]. Treatment sessions were held five times a week for two weeks (total: 10 sessions) [11]. Each session comprised 30 minutes of PES [37], with tDCS administered concomitantly during the first 20 minutes [21].

\section{Transcranial Direct Current Stimulation (tDCS)}

The one-channel DC Stimulation Plus (Neuroconn) device was used with two silicone/carbon electrodes measuring $5 \times 5 \mathrm{~cm}^{2}$ (both anode and cathode) covered with sponge soaked in $0.9 \%$ saline solution [38]. The anode was positioned over the motor cortex of the damaged hemisphere (C1 or C2) and the cathode was positioned over the motor cortex of the undamaged hemisphere (C1 or C2), both at a distance of $2 \mathrm{~cm}$ from $\mathrm{Cz}$ [22] on the map of the 10-20 International Electroencephalogram System[38]. Central stimulation with tDCS was administered with a current of $2 \mathrm{~mA}$ [11] during the first twenty minutes of PES. Sham stimulation involved the same procedures as active stimulation, but the device was activated only during the first 20 seconds and then the current was reduced to zero. All participants were informed that they could feel a slight tingling sensation that could disappear or remain during the 20 minutes of treatment [21]. This is considered a valid control procedure in studies involving tDCS [11].

\section{Determination of Potential Side Effects}

Possible side effects stemming from noninvasive brain stimulation were investigated using the TDCS - Side Effects Questionnaire (version translated into Portuguese) after each session [39].

\section{Peripheral Electrical Stimulation (PES)}

The two-channel QUARK® FES VIF 995 DUAL equipment was used for PES with two self-adhesive rubber electrodes measuring 5 $\mathrm{x} 9 \mathrm{~cm}$ positioned over the motor point and the belly of the paretic TA [21]. The PES parameters were pulse width of $250 \mu$ s, frequency of $50 \mathrm{~Hz}$, intensity increased until reaching the motor threshold (20-30\% of maximum voluntary contraction) and a 1:2 stimulation cycle (TON for $6 \mathrm{~s}$ and TOFF for $12 \mathrm{~s}$ ) [21] for 30 minutes [37]. The same parameters were used for sham PES, but the electrodes were positioned on the bone portion of the tibia [40].

\section{Statistics}

Descriptive statistics were performed for the characterization of the sample, distribution of the inferential data and comparison of the groups. The parametric variables (RMS and MDF) were compared using repeated-measures analysis of variance (ANOVA) (intragroup and intergroup analyses). The nonparametric variable (general balance score) was compared using either Friedman's test (intragroup analysis) or the Kruskal-Wallis test (intergroup analysis).

\section{Result}

Seventy-three volunteers were recruited for the present study. Thirty-seven were excluded for not meeting the eligibility criteria, two withdrew from the study for personal reasons before undergoing the evaluations and treatment, and one suffered an accident at home resulting in a hip fracture. Thus, 36 individuals were included in the study and randomized to the three intervention groups (Figure 1). Table 1 displays the demographic data of the 36 participants. The patients in the active PES + sham tDCS had hemiparesis 12 months longer than the other two groups. The active PES + active tDCS and sham PES + active tDCS groups had more patients with a diagnosis of hemorrhagic stroke than those in the active PES + sham tDCS group. All individuals in all three groups were classified with mild impairment based on the FMS for the lower limbs, but the active PES + active tDCS and sham PES + active tDCS groups has scores indicative of severe impairment for the overall FMS. The RMS and MDF were similar among the groups at baseline. Balance (evaluated using the Mini BesTest) was similar for the active PES + active tDCS and sham PES + active tDCS groups, whereas the active PES + sham tDCS group had somewhat higher scores. Table 1 . Characteristics of participants $(n=36)$. 


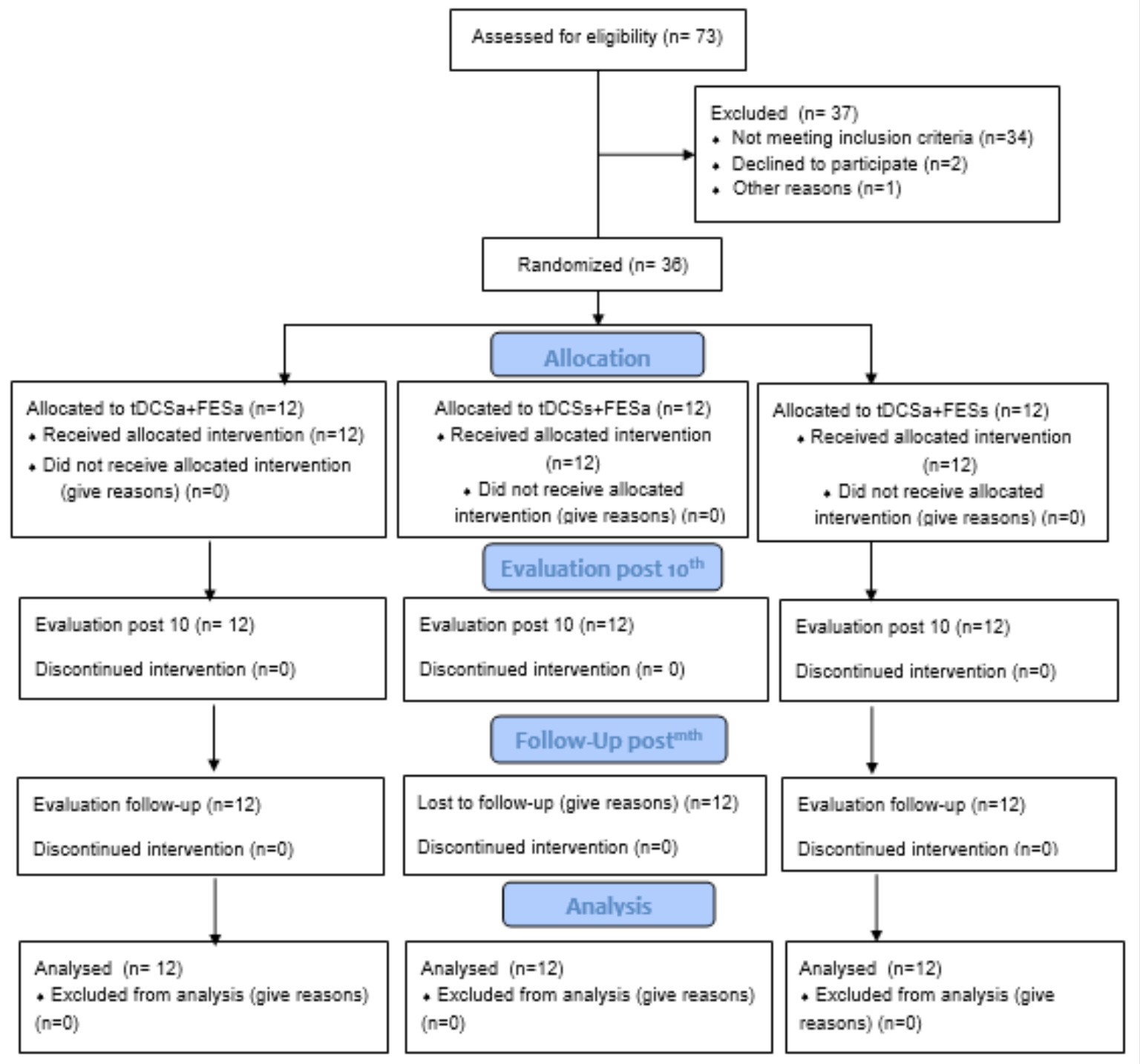

Figure 1: Flowchart of studsssy.

Table 1: Characteristics of participants $(n=36)$.

\begin{tabular}{|c|c|c|c|}
\hline Variables & Active PES/sham tDCS (n=36) & Active PES/active tDCS (n=36) & Sham PES/active tDCS (n=36) \\
\hline Gender(F/M) & $(6 / 6)$ & $(5 / 7)$ & $(7 / 5)$ \\
\hline Age (Years) & $62 \pm 9.59$ & $55.41 \pm 10.37$ & $59.25 \pm 10.48$ \\
\hline Stroke(H/I) & $(1 / 11)^{*}$ & $(4 / 8)$ & $(3 / 9)$ \\
\hline Right Hemisphere Affected & $(44.4 \%)$ & $(55.5 \%)$ & $(41.6 \%)$ \\
\hline Left Hemisphere Affected & $(55.5 \%)$ & $(44.4 \%)$ & $(58.3 \%)$ \\
\hline Time since Stroke (Months) & $60.91 \pm 76.61^{*}$ & $47.66 \pm 43.19$ & $47.0 \pm 27.17$ \\
\hline Antidepressant & $(8.33 \%)$ & $(16.6 \%) *$ & $(8.33 \%)$ \\
\hline ASA & $(0 \%)$ & $(0 \%)$ & $(8.33 \%)$ \\
\hline Diuretic & $(25 \%)^{*}$ & $(0 \%)$ & $(58 \%)$ \\
\hline Anti-Hypertensive & $(75 \%)^{*}$ & $(16.6 \%) *$ & $(25 \%)$ \\
\hline Diabetes Medication & $(58.3 \%)^{*}$ & $(41.66 \%)$ & $(58.3 \%)$ \\
\hline Cholesterol Medication & $(58.30 \%)$ & $(8.33 \%)$ & $(8.33 \%)$ \\
\hline Pain Medication & $(8.33 \%)$ & $10.83 \pm 7.77$ & $10.25 \pm 5.44$ \\
\hline BDI(Score) & $14.75 \pm 10.19$ & & \\
\hline
\end{tabular}




\begin{tabular}{|c|c|c|c|}
\hline MMSE(Score) & $24.91 \pm 2.99$ & $24.41 \pm 5.99$ & $23.33 \pm 4.92$ \\
\hline FMS (Score) & $72.66 \pm 25.95^{*}$ & $50.25 \pm 30.14$ & $48.41 \pm 26.26$ \\
\hline FMS-LL (Score) & $21.5 \pm 4.71$ & $23.14 \pm 9.02$ & $24.55 \pm 15.55$ \\
\hline AS M.Triceps Surais(Score) & $2[1-4]$ & $3[2-4]$ & $2[1-4]$ \\
\hline MS M.Tibialis Anterior (Score) & $3[1-4]$ & $3[2-3]$ & $3[2-4]$ \\
\hline RMS(Watt) & $0.12 \pm 0.02$ & $0.14 \pm 0.01$ & $0.14 \pm 0.02$ \\
\hline MDF(Hz) & $98.35 \pm 27.02$ & $99.05 \pm 26.19$ & $98.08 \pm 25.81$ \\
\hline MB (General Score) & $20.0[10.25-24]^{*}$ & $11.19[8.83-17.24]$ & $12.50[7.25-21.25]$ \\
\hline
\end{tabular}

Note: Data expressed as mean \pm standard deviation; median [interquartile range], percentage $(\%),(\mathrm{F} / \mathrm{M})(\mathrm{male} / \mathrm{female}),(\mathrm{H} / \mathrm{I})$ (hemorrhagic/ischemic), ASA (acetylsalicylic acid), BDI (Beck Depression Inventory), MMSE (Mini Mental State Examination), FMS (Fugl-Meyer Scale), FMS - LL (Fugl-Meyer Scale for lower limbs), AS (Ashworth Scale), MS (muscle strength), RMS (root mean square) in unit of measure (watt), MDF (median frequency) in unit of measure (Hz), MB (Mini BesTest), ${ }^{*} \mathrm{p}<0.05$ (repeated-measures ANOVA).

Figure 2 displays the results of the analysis of the amplitude of the electromyographic signals (RMS). No significant intra-group or inter-group differences were found at the post-intervention or follow-up evaluations ( $p>0.05$ ). The effect size was also nonsignificant for all groups (Cohen's $d<0.19$ ). Figure 2. Amplitude of the signal (root mean square [RMS] in watts) in the three groups at the three evaluation times. Figure 3 displays the results of the MDF of the electromyographic signals. In the intra-group analysis, a significant reduction in the MDF was found at the post-intervention evaluation in the active PES + sham tDCS group ( $p=0.04)$, but an increase found at the follow-up evaluation (reaching a higher value in comparison to baseline, although without achieving statistical

significance). A significant reduction was found at the postintervention evaluation $(p=0.02)$ and follow-up evaluation $(p=$ 0.04 ) in the active PES + active tDCS group. Significant reductions were also found at the post-intervention $(p=0.03)$ and follow-up ( $p=0.04$ ) evaluations in the sham PES + active tDCS group. The effect size was non-significant in all groups (Cohen's $d<0.19$ ). In the inter-group analysis, a significant difference was found in the active PES + sham tDCS $(p=0.00)$ at the follow-up evaluation (when the MDF returned to baseline values) in comparison to the active PES + active tDCS and sham PES + active tDCS groups. Figure 3 - Median frequency (MDF in $\mathrm{Hz}$ ) in the three groups at the three evaluation times. *p $<0.05$ (repeated-measures ANOVA).

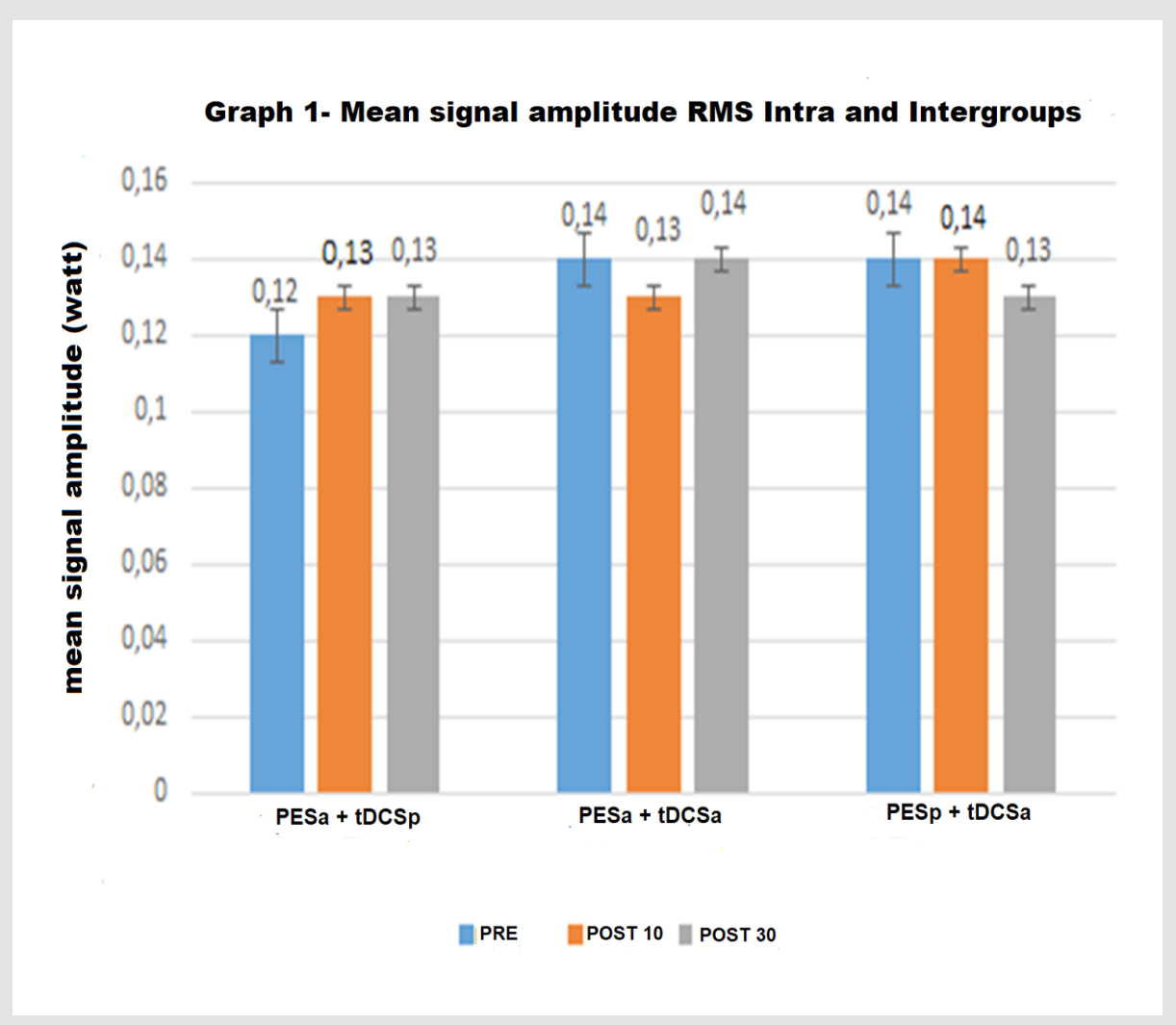

Figure 2: Amplitude of the signal (root mean square [RMS] in watts) in the three groups at the three evaluation times. 


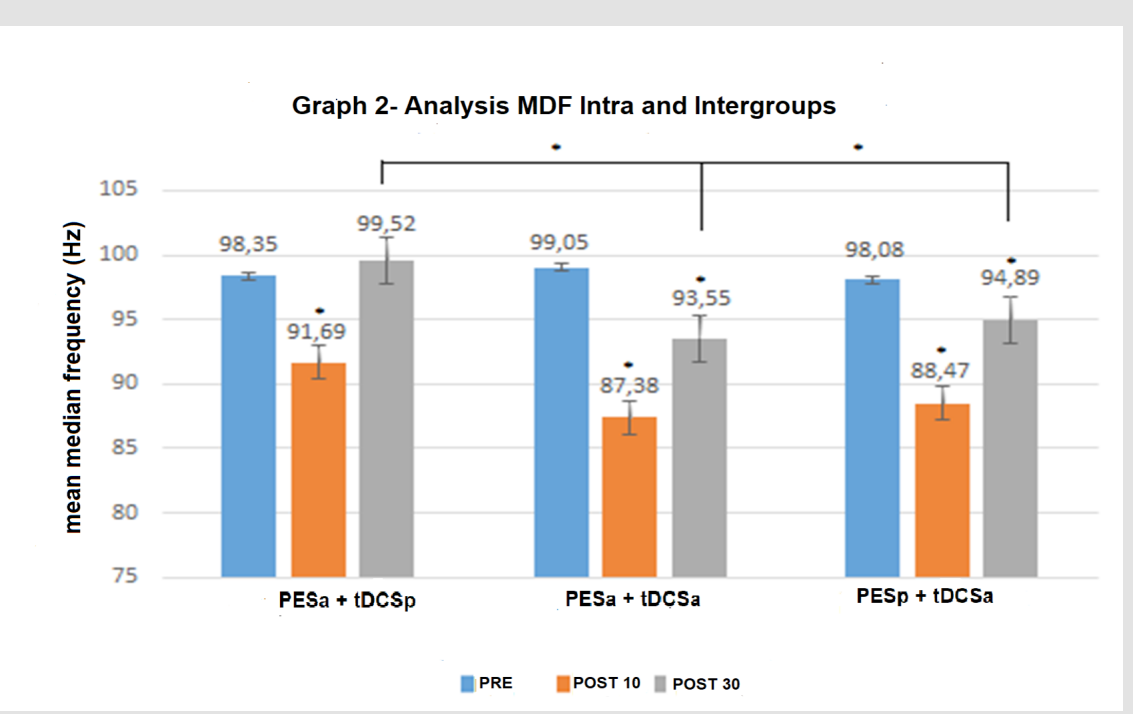

Figure 3: Median frequency (MDF in $\mathrm{Hz}$ ) in the three groups at the three evaluation times. * $\mathrm{p}<0.05$ (repeated-measures ANOVA).

Figure 4 displays the balance results (overall Mini BES Test). In the intra-group analysis, a statistically significant improvement was found at the post-intervention and follow-up evaluations only in the active PES + active tDCS group ( $p=0.00)$. No significant inter-group differences were found ( $p>0.05$ ). However, a clinically important change ( $>3$ points $)^{35}$ was found in all groups: active PES + sham tDCS at post-intervention evaluation (3.5); active PES + active tDCS at post-intervention evaluation (10.31) and follow-up (8.31); sham PES + active tDCS at post-intervention evaluation (5.5) and follow-up (3.5). These findings demonstrate an improvement in balance. The effect size was non-significant in all groups (Cohen's $d<0.19$ ). Figure 4-Results of Mini BES Test (overall score: 0 to 28 points) in the three groups at the three evaluation times. ${ }^{*} \mathrm{p}<0.05$ (Friedman's test).

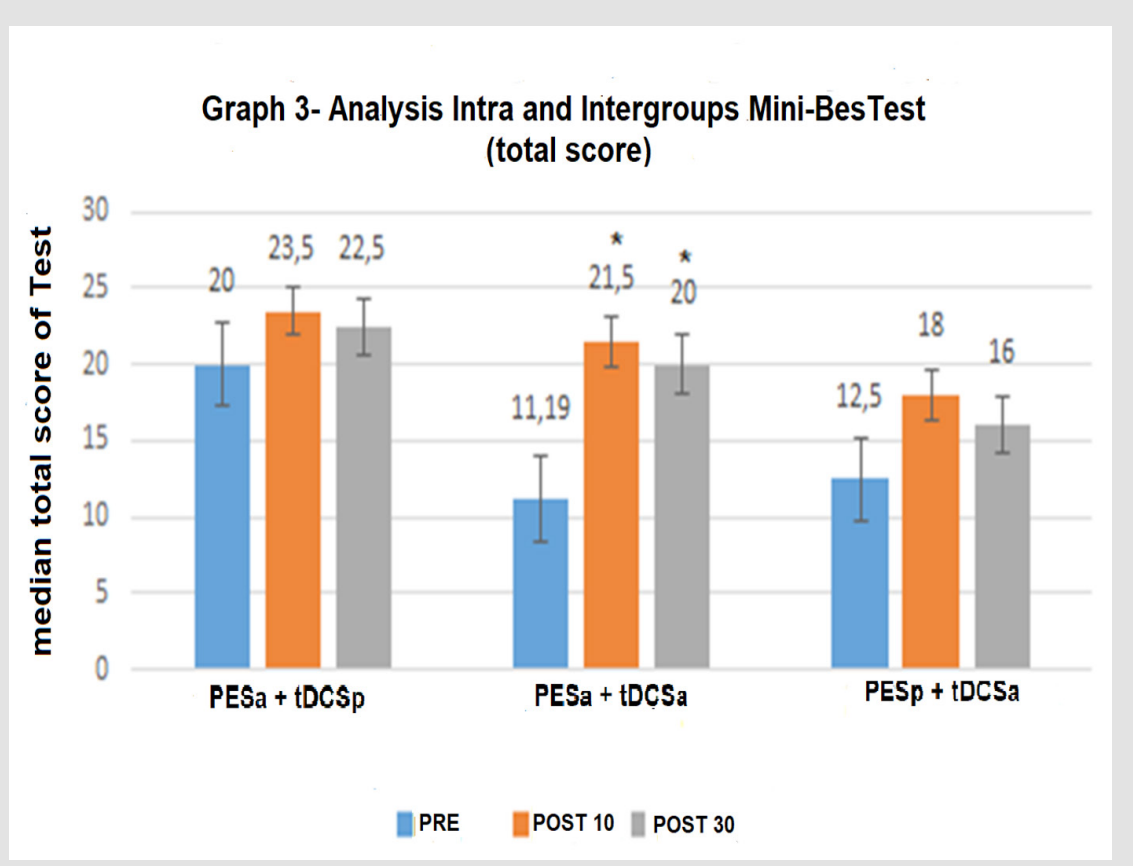

Figure 4: Results of Mini BESTest (overall score: 0 to 28 points) in the three groups at the three evaluation times. * $\mathrm{p}<0.05$ (Friedman's test).

\section{Discussion}

\section{Electrical Activity of TA Muscle}

No significant or clinically important intra-group or inter-group differences in RMS were found at either the post- intervention evaluation (after ten treatment sessions) or the follow-up evaluation (30 days after the end of the interventions). Thus, central stimulation combined with peripheral stimulation was not effective regarding the electrical activity of the TA muscle, even when sham PES was performed. The literature reports 
that resistance training with maximum load (determined in an individualized manner) is needed to achieve an increase in power [41]. We may therefore suppose that the lack of such training may explain the non-occurrence of an increase in the amplitude of the electromyographic signals of the muscle fibers, which hindered the intrinsic action of central and peripheral stimulation.

Regarding the MDF, a significant reduction was found after ten treatment sessions in all groups, which was maintained at the follow-up evaluation in the active PES + active tDCS and sham PES + active tDCS groups. Thus, rather than enhancing the information stemming from peripheral stimulation, central stimulation (tDCS) may have provoked the inverse effect - inhibiting it. This suggestion is based on the theory of cerebral homeostasis put forth by Bienenstock et al. [42], who postulates that the threshold for inducing long-term potentiation (LTP) or long-term depression (LTD) is not constant, but varies in accordance with the level of activity of the post-synaptic neuron. A high level of synaptic excitability may subsequently reduce any associated task that also requires a recurrent increase in neuronal activity triggered by an neurophysiological defense. Fricke et al. [43] report that long periods of tDCS (greater than $10 \mathrm{~min}$ ) with no break in the first five minutes can lead to a reduction in cortical excitability. Therefore, we believe that a set of factors related to the characteristics of the population investigated triggered the homeostatic defense discovered by Bienenstock. The participants in this study were in the chronic phase of stroke and previous studies [44] have proved that a long-term injury to the nervous system often leads to greater resistance of the neuronal membrane to information subsequently imposed on the system. We also believe that a sedentary lifestyle and long periods without any type of treatment, as occurred in the present study, makes the nervous system consider any motor task (regardless of its complexity) to be a high excitability threshold, which forces a blocking of its actions.

Thus, we did not confirm the hypothesis from the first study of our research group [21], in which we suppose that the combination of central and peripheral stimulation did not achieve positive results because only a single treatment session was used. A negative result regardless of the duration of treatment was also reported in the study by Rizzo et al. [18], who found a reduction in the MEP in healthy individuals after 10, 20, 30 and 60 minutes of tDCS over M1 combined with repetitive electrical stimulation of the median nerve. However, these findings differ from those reported by Madhavan et al. [13], who found an increase in MEP for 15 minutes and immediately after the practice of ankle dorsiflexion training combined with tDCS over M1 in stroke survivors. Likewise, Sriraman et al. [45] found an increase in the MEP in healthy adults before, during (20 minutes) and 24 hours after tDCS administered over M1 combined with a visual ankle motor task. Ziemann et al. [46] report that including tDCS only in some periods of a motor task may enhance the learning of this task, thus deviating from mechanisms of metaplasticity. Therefore, a different protocol from that used in the present study regarding the duration of the combination of central and peripheral stimulation may lead to different results.

No change in RMS or increase in MDF occurred in the active PES + sham tDCS group. The amount of stimulation (10 sessions) may not have been sufficient to increase the activity of the TA muscle, as previous studies with a longer PES time report positive effects on the activation of this muscle or a functional improvement in ankle movement. Newsam et al. [47] found a significant improvement in the recruitment of motor units following PES to the TA muscle applied five times a week for three weeks. Knutson et al. [48] investigated the effects of PES five times a week for six weeks on active dorsiflexion of the angle and found a significant increase in the range of motion of this joint. Mesci et al. [49] found a significant increase in ankle dorsiflexion and a reduction in spasticity after four weeks of treatment with PES.A more concrete explanation for the present results may be achieved through neurophysiological analyses or the quantification of the torque of the TA muscle. When torque increases, the electrical activity of the muscle tends to decrease. The lack of such analyses is one of the limitations of the present study.

\section{Functional Balance}

A statistically significant improvement on the Mini-BES Test was only found in the active PES + active tDCS group. However, a clinically important improvement in balance ( $>3$ points) was found in all groups. According to Potter et al. [31], a three-or-more-point increase in the overall score predicts a minimum detectable change (MDC) for the test. These findings are in agreement with data reported in previous studies on balance outcomes in patients with neurological diseases submitted to tDCS combined with other motor tasks. Kaski et al. [50] found improvements in balance and gait in individuals with leukoaraiosis (white matter disease) following a single session of tDCS combined with physical training. Analyzing 24 children aged five to 12 years with cerebral palsy, Duarte et al. [51] found a significant improvement in the Pediatric Balance Scale as well as both anteroposterior (eyes open and closed) and laterolateral (eyes closed) sway following tDCS combined with treadmill training both one week and one month after the conclusion of the intervention. Dumont et al. [52] evaluated static balance in a stroke survivor after a single session of tDCS over M1 combined with treadmill training and found reductions in anteroposterior sway as well as oscillation area and velocity.

The clinical improvement in the sham PES + active tDCS group was lower than that found in the active PES + active tDCS group at the post-intervention and follow-up evaluations. This confirms reports in the literature of greater effectiveness when tDCS is combined with other therapies [53]. However, Sohn et al. [14] report positive results in terms of functioning after tDCS administered over the damaged primary motor cortex without using a concomitant motor task. The authors found a significant improvement in postural 
stability and an increase in the strength of the quadriceps muscle in 11 stroke survivors with hemiparesis. In the active PES + sham tDCS group, a clinically important improvement was found at the post-intervention evaluation. This finding is in agreement with data described in a study by Hyun et al. [54], who applied functional electrical stimulation (FES) to the paretic TA muscle in stroke survivors and found significant improvements in both balance and quality of life. We believe that the sensorimotor stimuli promoted by PES in the present study were able to activate intact fibers of the pyramidal tract, [55] consequently favoring the adjustment of postural control but without actually inducing actions related to mechanisms of neuroplasticity.

The present results suggest that the effects found on balance are not directly related to motor training of the TA muscle, as neither the RMS nor MDF improved in the groups. Thus, the effects may be linked to cortical afferences, which modulated neuronal excitability for the lower limbs [56]. Another possibility is that other areas of the central nervous system involved in balance may have been activated, as stimulus with tDCS is not merely localized. Indeed, Leonor et al. [57] report that electrical stimuli from tDCS can reach the tracts underlying the stimulated tract as well as primary and secondary motor areas. However, the mechanisms behind this remain unclear. The fact that an improvement in balance occurred independently of the improvement in the electrical activity of the TA muscle demonstrates that tDCS may have activated important structures in the central nervous system responsible for postural control and, therefore, the isolated action of the TA muscle did not exert an impact on this motor task.

\section{Conclusion}

In the present study, central stimulation with tDCS did not enhance the effects of peripheral stimulation regarding the electrical activity of the tibialis anterior and actually led to a reduction in the activity of the muscle. However, different results were found regarding balance, as the combination of central and peripheral stimulation led to a clinically important improvement in this variable. Thus, tDCS can assist in complex motor tasks, such as postural balance.

\section{Acknowledgment}

The authors acknowledge Nove de Julho University for supporting the present study.

\section{Authors' Contributions}

FIC and AMAF designed the study. CCS and DCA performed the data collection, interventions and recruitment of the participants. FIC and AMAF prepared the manuscript, which was revised and edited by FP and JCF. All authors read and approved the final manuscript.

\section{Competing interests}

The authors declare that they have no competing interests.

\section{Trial Registration}

ClinicalTrials.gov - NCT03008720.

\section{Ethical Approval}

This protocol received approval from the Human Research Ethics Committee of Nove de Julho University, São Paulo, Brazil (certificate number: 2.015.168) in compliance with Resolution $466 / 12$ of the Brazilian National Board of Health.

\section{References}

1. Mayer NH, Esquenazi A (2003) Muscle overactivity and movement dysfunction in the upper motoneuron syndrome, Phys Med Rehabil Clin N Am 14(4): 855-883

2. Lance JW (1980) The control of muscle tone, reflexes, and movement: Robert Wartenberg Lecture. Neurology 30(12): 1303-1313.

3. Brandalize D, Brandalize M (2010) Efeitos da toxina botulínica no tratamento do pé eqüino em indivíduos com seqüelas de acidente vascular encefálico. VOOS Revista Polidisciplinar Eletrônica da Faculdade Guairacá: 3-13.

4. Pérennou D (2005) Weight bearing asymmetry in standing hemiparetic patients. J Neurol Neurosurg Psychiatry 76(5): 621.

5. Ferreira LAB, Rossib LP, Pereira WM, Vieira FF, Paula ARDJ (2009) Análise da Atividade Eletromiográfica dos Músculos do Tornozelo em solo estável e instável. Fisioter Mov pp. 177-187.

6. Perry J (2005) Análise da Marcha: marcha patológica. Barueri, Manole, Bulgaria.

7. Rushton DN (2003) Functional Electrical Stimulation and rehabilitationA hypothesis. Medical Engineering \& Physics: 75-78

8. Anel H, Weingarden $\mathrm{H}$ (2007) Neuromodulation by functional electrical stimulation (FES) of limb paralysis after stroke. Acta. Neurochir pp. 375380.

9. Lucarelli PRG (2005) Avaliação das atividades de vida diária pelo Índice de Barthel de pacientes acometidos de acidente vascular encefálico. Fisioter Bras, São Paulo 6(2): 109-112.

10.Schlaug G, Renga V, Nair D (2008) Transcranial Direct Current Stimulation in Stroke Recovery. Arch Neurol 65(12): 1571-1576.

11. Adeyemo BO, Simis M, Macea DD, Fregni F (2012) Systematic review of parameters of stimulation, clinical trial design characteristics, and motor outcomes in non-invasive brain stimulation in stroke. Front Psychiatry 3: 88.

12. Rahman A, Reato D, Arlotti M, Gasca F, Datta A, et al. (2013) Cellular effects of acute direct current stimulation: somatic and synaptic terminal effects. J Physiol 591(10): 2563-2578.

13. Madhavan S, Weber KA, Stinear JW (2011) Non-invasive brain stimulation enhances Wne motor control of the hemiparetic ankle: implications for rehabilitation. Exp Brain Res p. 9-17.

14. Sohn MK, Sung JJ, Yeong W (2013) Effect of Transcranial Direct Current Stimulation on Postural Stability and Lower Extremity Strength in Hemiplegic Stroke Patients. Ann Rehabil Med pp. 759-765.

15. Nyeonju K, Jeffery JS, James HC (2015) Transcranial direct current stimulation facilitates motor learning post-stroke: a systematic review and meta-analysis. J Neurol Neurosurg Psychiatry p. 1-11.

16. Lindenberg R, Zhu LL, Schlaug G (2012) Combined Central and Peripheral Stimulation to Facilitate Motor Recovery After Stroke: The Effect of Number of Sessions on Outcome. Neurorehabilitation and Neural Repair 26(5): 479-483.

17. Kwon, Jung Won Kwon, Sang Young Park, Sung Ho Jang (2011) Cortical Activation by Transcranial Direct Current Stimulation and Functional Electrical Stimulation in Normal Subjects: 2 Case Studies. J Kor Soc Phys Ther 23(1): 77-82. 
18. Rizzo V, Terranova C, Crupi D, Santangelo A, Girlanda P, et al. (2014) Increased transcranial direct current stimulation aftereffects during concurrent peripheral electrical nervestimulation. Brain Stimul pp. 113211.

19. Celnik P, Paik NJ, Vandermeeren Y, Dimyan M, Cohen LG (2009) Effects of combined peripheral nerve stimulation and brain polarization on performance of a motor sequence task after chronic stroke. Stroke 40(5): 1764-1771.

20. Sattler V, Acket B, Raposo N, Albucher JF, Thalamas C, et al. (2015) Anodal tDCS Combined with Radial Nerve Stimulation Promotes Hand Motor Recovery in the Acute Phase After Ischemic Stroke, Neurorehabilitation and Neural Repair 29(8): 743-754.

21. Fruhauf AMA, Politti F, Dal Corso S, Costa GC, Teodósio ADC, et al. (2017) Immediate effect of transcranial direct current stimulation combined with functional electrical stimulation on activity of the tibialis anterior muscle and balance of individuals with hemiparesis stemming from a stroke. J Phys Ther Sci 29(12): 2138-2146.

22. Fruhauf, Evaluating Electrical Activity of Tibialis Anterior Muscle and Balance in Hemiparetic Patients Following Central and Peripheral Electrical Stimulation - Protocol for a Randomized, Double-blinded, Clinical Trial.

23. Hermans G, Clerckx B, Vanhullebusch T, Segers J, Vanpee G, et al. (2012) Interobserver agreement of Medical Research Council sumscore and handgrip strength in the intensive care unit. Muscle Nerve 45(1): 18-25.

24. Maheshwari SG, IqbaL M, Hashmi SFA, Devrajani B (2015) Stroke patients; assessment of cognitive impairment in. Professional Med J pp. 541- 545 .

25. Beck AT, Ward CH, Mendelson M, Mock J, Erbaugh J (1961) An inventory for measuring depression. Arch. Gen. Psychiatry 4: 561-571.

26. Ashword B (1964) Preliminary trial of carisoprodol in multiple sclerosis. Practitioner, pp. 540-542.

27. Sabut R, Kumar PK, Lenka MM (2010) Surface EMG Analysis of Tibialis Anterior Muscle in Walking with FES in Stroke Subjects. Conf Proc IEEE Eng Med Biol Soc pp. 5839-5842.

28. Hermens HJ, Freriks B, Disselhorst Klug C, Rau G (2000) Development of recommendations for SEMG sensors and sensor placement procedures. J. Electromyography and Kinesiology pp. 361-374.

29. Suhaimi R, Nazrul H Adnan, Fakhrul Asyraf, Wan Khairunizam (2014) Analysis of EMG-based Muscles Activity for Stroke Rehabilitation. $2^{\text {nd }}$ International Conference on Electronic Design, ICED.

30. Tania FC (2010) Electromyographic analysis of spastic muscles in hemiparetic patients before and after physical therapy intervention. Ter Man 10(48): 148-153.

31. Potter, Kirsten (2015) The Mini-Balance Evaluation Systems Test (MiniBESTest). Journal of Physiotherapy 61(4): 225.

32. Charlotte SLT, Lin RL, Raymond CK, Chung MYC (2013) Psychometric Properties of the Mini-Balance Evaluation Systems Test (Mini-BESTest) in Community-Dwelling Individuals with Chronic Stroke. Phys Ther 93 (8): 1102-1115.

33. Kroenke K, Spitzer RL, Williams JB (2001) The PHQ-9: validity of a brief depression severity measure. J Gen Intern Med 16(9): 606-613.

34. An TG, Kim SH, Kim KU (2017) Effect of transcranial direct current stimulation of stroke 528 patients on depression and quality of life. J Phys Ther Sci 29(3): 505-507.

35. Fugl Meyer AR, Jaasko L, Leyman I, Olsson S, Steglind S (1975) The post-stroke hemiplegic patient: A method for evaluation of physical performance. Scand. J Rehab Med p. 13-31.

36. Maki T, Quagliato Emab, Cacho Ewa, Paz Lps, Nascimento Nh, et al. (2006) Estudo de confiabilidade da aplicação da escala de fugl-meyer no Brasil. Rev Bras Fisioter 10(2): 177-183.
37. Howlett OA, Lannin NA, Ada L, Mckinstry C (2015) Functional electrical stimulation improves activity after stroke: a systematic review with meta-analysis. Arch Phys Med Rehabil pp. 934-943.

38. Dasilva AF, Volz MS, Bikson M, Fregni F (2011) Electrode Positioning and Montage in Transcranial Direct Current Stimulation. J Vis Exp p. 1-11.

39. Xu J, Fregni F, Brody AL, Rahman AS (2013) Transcranial direct current stimulation reduces negative affect but not cigarette craving in overnight abstinent smokers. Front Psychiatry 20: 4-12.

40. Ambrosini E, Ferrante S, Pedrocchi A, Ferrigno G, Molteni F (2011) Cycling Induced by Electrical Stimulation Improves Motor Recovery in Postacute Hemiparetic Patients: A Randomized Controlled Trial. Stroke 42: 1068-1073.

41. Aline Rodrigues Barbosa, José Maria Santarém, Wilson Jacob Filho, Maria de Fátima Nunes Marucci (2000) Efeitos de um programa de treinamento contra resistência sobre a força muscular de mulheres idosas. Revista Brasileira de Atividade Física e Saúde 5(3): 12-20.

42. Bienenstock EL, Cooper LN, Munro PW (1982) Theory for the development of neuron selectivity: orientation specificity and binocular interaction in visual cortex. J Neurosci p. 32-48.

43. Fricke K, Seeber AA, Thirugnanasambandam N, Paulus W, Nitsche MA, et al. (2013) Time course of the induction of homeostatic plasticity generated by repeated transcranial direct current stimulation of the human motor cortex. J Neurophysiol 105: 1141-1149.

44. Madhavan S, Rogers LM, Stinear JW (2010) A paradox: After stroke, the non-lesioned lower limb motor cortex may be maladaptive. Eur J Neurosci 32: 1032-1039.

45. Sriramana A, Oishib T, Madhavan S (2014) Timing-dependent priming effects of tDCS on ankle motor skill learning. Brain Res p. 2-7.

46. Ziemann U, Siebner HR (2008) Modifying motor learning through gating and homeostatic metaplasticity. Brain stimulation 1: 60-66.

47. Newsam CJ, Baker LL (2004) Effect of na Electric Stimulation Facilitation Program on Quadriceps Motor Unit Recruitment After Stroke. Arch Phys Med Rehabil pp. 2040-2045.

48. Knutson JS, Chae J (2010) A novel neuromuscular electrical stimulation treatment for recovery of ankle dorsiflexion in chronic hemiplegia: a case series pilot study. Am J Phys Med Rehabil pp. 672-682.

49. Mesci N, Ozdenir F, Kabayel DD, Tokuc B (2009) The effects of neuromuscular electrical stimulation on clinical improvement in hemiplegic lower extremity rehabilitation in chronic stroke: a singleblind, randomized, controlled trial. Disabil. Rehabil pp. 2047-2054.

50. Kaski D, Dominguez RO, Allum JH, Bronstein AM (2013) Improving Gait and Balance in Patients With Leukoaraiosis Using Transcranial Direct Current Stimulation and Physical Training An Exploratory Study. Neurorehabilitation and neural repair pp. 864-871.

51. Duarte NA, Grecco LAC, Galli M, Fregni F, Oliveira CS (2014) Effect of transcranial direct-current stimulation combined with treadmill training on balance and functional performance in children with cerebral palsy: a double-blind randomized controlled trial. PloS one.

52. Dumont AJ, Araujo MC, Lazzari RD, Santos CA, Carvalho DB, et al. (2015) Effects of a single session of transcranial direct current stimulation on static balance in a patient with hemiparesis: A case study. J Phys Ther Sci.

53. Schlaug G, Renga V, Nair D (2008) Transcranial Direct Current Stimulation in Stroke Recovery. Arch Neurol 65(12): 1571-1576.

54. Hyun J, KYU L, Uk J, Young M, Yang SM, et al. (2013) A pilot study on the effect of functional electrical stimulation of stroke patients in a sitting position on balance and activities of daily living. J Phys Ther Sci pp. 1097-1101

55. Letícia E, Andressa PSS, Marijose PN, Heloisa V, Maurício C, et al. (2007) Os efeitos da Estimulação Elétrica Funcional na Assimetria Cortical Inter-Hemisférica. Arq Neuropsiquiatr 65(3-A): 642-646. 
56. Chang MC, Kim DY, Park DH (2015) Enhancement of Cortical Excitability and Lower Limb Motor Function in Patients with Stroke by Transcranial Direct Current Stimulation. Brain Stimulation 8: 561-556.

\section{ISSN: 2574-1241}

DOI: $10.26717 /$ BJSTR.2020.25.004219

Aline Marina Alves Fruhauf. Biomed J Sci \& Tech Res

cC) This work is licensed under Creative

Submission Link: https://biomedres.us/submit-manuscript.php
57. Romero Lauro LJ, Rosanova M, Mattavelli G, Convento S, Pisoni A, et al. (2016) TDCS increases cortical excitability: Direct evidence from TMSeEEG. Cortex 74: 320-322.

$\begin{array}{ll}\text { BIOMEDICAL } & \text { Assets of Publishing with us } \\ \text { RESEARCHES } & \text { - Global archiving of articles } \\ & \text { - Immediate, unrestricted online access } \\ & \text { - Rigorous Peer Review Process } \\ & \text { - Authors Retain Copyrights } \\ \end{array}$

